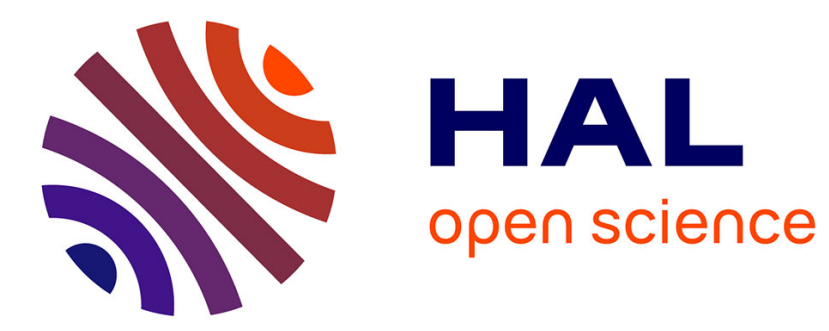

\title{
La scolarisation des patients atteints par des Troubles du Spectre Autistique sans retard mental \\ Dévi Grimm
}

\section{To cite this version:}

Dévi Grimm. La scolarisation des patients atteints par des Troubles du Spectre Autistique sans retard mental. Médecine humaine et pathologie. 2014. dumas-01063693

\section{HAL Id: dumas-01063693 https://dumas.ccsd.cnrs.fr/dumas-01063693}

Submitted on 12 Sep 2014

HAL is a multi-disciplinary open access archive for the deposit and dissemination of scientific research documents, whether they are published or not. The documents may come from teaching and research institutions in France or abroad, or from public or private research centers.
L'archive ouverte pluridisciplinaire HAL, est destinée au dépôt et à la diffusion de documents scientifiques de niveau recherche, publiés ou non, émanant des établissements d'enseignement et de recherche français ou étrangers, des laboratoires publics ou privés. 


\section{Université
Joseph Fourier MEDECINE}

\section{SICD1 \\ Bibliothèques \\ de I'UJF et de Grenoble INP}

\section{AVERTISSEMENT}

Ce document est le fruit d'un long travail approuvé par le jury de soutenance et mis à disposition de l'ensemble de la communauté universitaire élargie.

Il n'a pas été réévalué depuis la date de soutenance.

Il est soumis à la propriété intellectuelle de l'auteur. Ceci implique une obligation de citation et de référencement lors de l'utilisation de ce document.

D'autre part, toute contrefaçon, plagiat, reproduction illicite encourt une poursuite pénale.

Contact au SICD1 de Grenoble : thesebum@ujf-grenoble.fr

\section{LIENS}

Code de la Propriété Intellectuelle. articles L 122. 4

Code de la Propriété Intellectuelle. articles L 335.2- L 335.10

http://www.cfcopies.com/V2/leg/leg_droi.php

http://www.culture.gouv.fr/culture/infos-pratiques/droits/protection.htm 
UNIVERSITÉ JOSEPH FOURIER

FACULTÉ DE MÉDECINE DE GRENOBLE

Année : 2014

\section{La scolarisation des patients atteints par des Troubles du Spectre}

Autistique sans retard mental

THÈSE

PRÉSENTÉE POUR L'OBTENTION DU DOCTORAT EN MÉDECINE

DIPLÔME D'ÉTAT

Par Dévi GRIMM

Née le 14 Juin 1987 à Niamey (Niger)

Présentée et soutenue publiquement à la faculté de Médecine de Grenoble

Le 4 septembre 2014

Devant le Jury composé de :

Président du jury : Monsieur le Professeur Thierry BOUGEROL (psychiatrie)

\section{$\underline{\text { Membres : }}$}

Monsieur le Professeur Mircea POLOSAN (psychiatrie)

Monsieur le Professeur Pierre-Simon JOUK (génétique)

Monsieur le Docteur Andrea PIERO (psychiatrie)

Madame le Docteur Brigitte ASSOULINE (psychiatrie)

*La Faculté de Médecine de Grenoble n'entend donner aucune approbation ni improbation aux opinions émises dans les thèses; ces opinions sont considérées comme propres à leurs auteurs. 
A Monsieur le Professeur Thierry BOUGEROL, d'avoir accepté de présider mon jury de thèse, et pour ses conseils durant l'ensemble de mon internat, ainsi qu'à Gisèle BOUGEROL et Lilia MAAMAR.

A Monsieur le Professeur Mircea POLOSAN, d'avoir accepté de siéger dans mon jury et d'être présent auprès des internes.

A Monsieur le Professeur Pierre-Simon JOUK, d'avoir accepté de siéger dans mon jury, et pour l'aide qu'il apporte aux internes de psychiatrie en nous acceptant dans ses consultations.

Au Docteur Brigitte ASSOULINE, de m'avoir confié cette étude, de m'avoir fait confiance, pour sa disponibilité et son aide.

Au Docteur Andrea PIERO, pour sa patience lors des mes débuts, de m'avoir fait profiter de son expérience et de son aide pour la publication de cet article.

A Caroline, pour son aide précieuse durant l'élaboration et la correction de cet article, pour sa bonne humeur et pour son optimisme sans faille.

$\mathrm{Au}$ Docteur Jean-Paul CHABANNES, qui m'a toujours impressionnée et beaucoup appris, pour son implication et la formation fondamentale qu'il propose aux internes.

A tous les médecins qui m'ont suivie et formée durant mes stages, Olfa, Jérôme, Gaëlle, Elsa, Brigitte, Lise, Pierre, Yves, Juliette, Elisabeth, Félicie, Mouna, Faouzi, Vincent, Diana.

Et à toutes leurs équipes respectives qui ont également participé à ma formation, mes réflexions, qui m'ont fait évoluer et grandir.

A l'ensemble de mes co-internes.

A ma famille, mon père, ma mère, mes frères pour leur soutien durant mes nombreuses années d'études.

A Jean-Paul et Yveline, pour m'avoir toujours accueillie et considérée comme faisant partie de leur famille.

Et pour finir à Thomas, pour son amour, son aide, son soutien et tout le bonheur qu'il m'apporte chaque jour. 
1. HISTORIQUE ...........................................................................................................

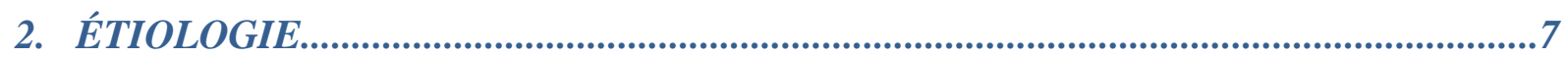

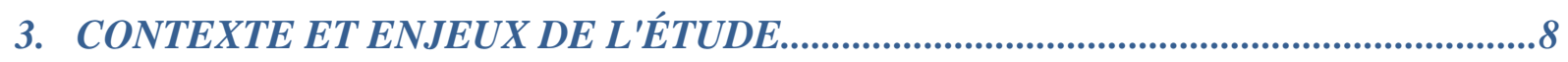

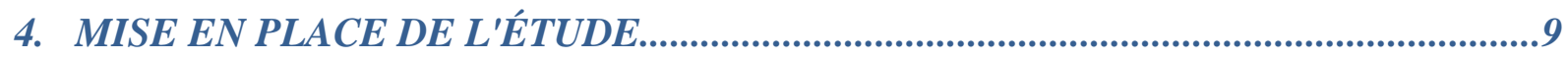

5. VALORISATION DE L'ÉTUDE ET PERSPECTIVES............................................11

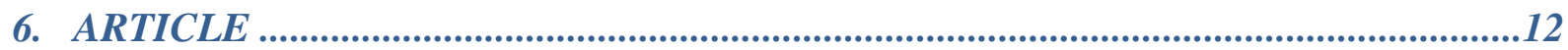

Résumé en français....................................................................................................................13

Résumé en anglais..........................................................................................................14

Introduction .....................................................................................................

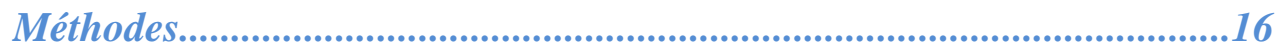

Résultats...........................................................................................18

Discussion..................................................................................19

Tableaux..........................................................................23

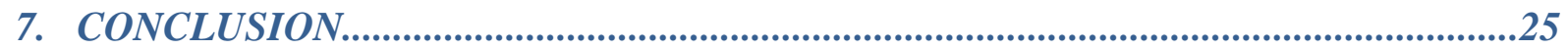

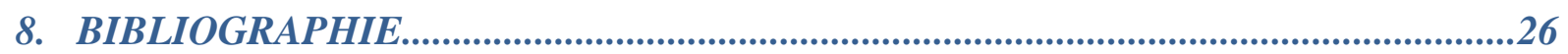

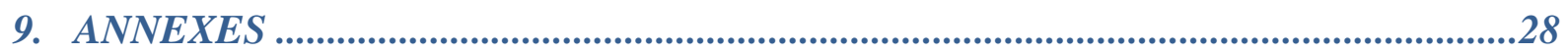

1. Classification de l'autisme selon le DSM-IV-TR .........................................................29

2. Classification de l'autisme selon la CIM-10 ...........................................................31

3. Classification de l'autisme selon le DSM-V .........................................................32

4. Premier courrier de réponse des réferees de l'Encéphale..........................34

5. Deuxième courrier d'acceptation de l'Encéphale.............................38

6. Récépissé de déclaration à la CNIL ........................................39

7. Exemple d'item de l'ADOS..............................................40

8. Exemple d'item de l'ADI-R....................................41

9. Exemples d'items du WISC-IV..............42

10. Serment d'Hippocrate..........................43 


\section{HISTORIQUE}

Le mot "autisme" est dérivé du grec "autos" qui signifie "soi-même".

Il est coutume en France de faire débuter l'histoire de l'autisme par la découverte de Victor, en 1798 dans l'Aveyron. Enfant d'une douzaine d'année, il a été retrouvé dans les bois de Lacaune, vivant à l'état sauvage. Victor ne pouvait pas parler et avait un comportement décrit comme sauvage, proche de l'animal. Il fut confié à Jean-Marc Gaspard Itard. Ce dernier conclu que les troubles de Victor étaient causés par le manque de civilisation qu'il avait subit. Il entreprit de l'instruire pendant 5 ans en démontrant que l'on pouvait rendre le comportement de Victor plus sociable et qu'il était possible de lui apprendre les rudiments du langage.

En 1846, on retrouve dans les publications d'Edouard Seguin (instituteur) sur "l'idiotisme" et les "enfants idiots", des symptômes étrangement similaires à ceux que Leo Kanner décrira plus tard : mutisme, incapacité de direction ou de fixité du regard, absence partielle ou totale de la faculté d'écouter, insensibilité tactile ou excès de sensibilité tactile, atonie, excès d'activité, mouvements mécaniques, balancement, dépravation du goût et de l'odorat.

En 1911, Eugen Bleuler utilise pour la première fois le terme "autisme" pour décrire le repli sur soi et sur le monde intérieur des patients atteints de schizophrénie.

Mais ce n'est qu'en 1943 à Baltimore, que Leo Kanner, psychiatre américain d'origine autrichienne, donne naissance à une nouvelle entité clinique qu'il nomme "autisme infantile précoce", distincte de l'arriération mentale. Il la considère comme une affection spécifique de l'enfance. Leo Kanner décrit dans ses articles les comportements d'enfants ayant une inaptitude à établir des relations normales avec autrui et à réagir normalement aux situations, depuis le début de leur vie. Il décrira lors de son rapport princeps en 1943 une certaine "typologie" des premières familles rencontrées, en introduisant la dénomination de "refrigerator mother". Ces descriptions entraineront par la suite de nombreuses controverses. 
L'autisme est alors rapidement reconnu comme une affection autonome, la distinguant des formes précoces de schizophrénie.

La même année, le pédiatre viennois Hans Asperger décrit "les psychopathies autistiques de l'enfance". Ses travaux ne seront repris qu'en 1981 par Lorna Wing, psychiatre anglaise, qui utilisera pour la première fois le terme de Syndrome d'Asperger (SA).

La clinique de l'autisme précoce infantile regroupe alors :

\section{Retrait autistique:}

- Peu ou pas de contact avec la réalité externe (l'enfant se comporte comme si autrui n'existait pas)

- Crainte du contact corporel

- Utilisation d'une partie du corps de l'autre comme objet ou comme prolongation de son propre corps

\section{Besoins d'immuabilité :}

- Besoin d'un environnement stable et inchangé

- Conduites ritualisées de vérification

\section{Stéréotypies:}

- Gestes répétés : balancement du tronc, flapping, tapotage, tournoiement...

\section{Trouble du langage variable:}

- Retard de langage

- Difficulté à utiliser le langage dans un but de communication

- Inversion pronominale (l'enfant parle de lui à la $2 \mathrm{e}$ ou $3 \mathrm{e}$ personne)

- Echolalies

\section{Début très précoce}




\section{Nosographie:}

De nombreux débats et controverses sur la classification de l'autisme ont eu lieu.

En 1980 pour le DSM (Diagnostic and Statistical Manual of Mental Disorders) et en 1986 pour la CIM (Classification Internationale des Maladies), les "psychoses infantiles" disparaissent. Elles laissent place aux Troubles Envahissants du Développement (TED) dont fera partie l'autisme.

Lors de sa révision en 1987, le DSM-III distingue deux catégories de TED : les troubles autistiques et les TED non spécifiés.

La dixième édition de la CIM introduit les catégories "autisme atypique" et "syndrome d'Asperger".

En 1994, le DSM-IV (Annexe 1) s'aligne sur les critères de la CIM-10 (Annexe 2). Dans le récent DSM-V (traduction en cours), l'autisme est désormais décrit selon un mode dimensionnel et prend le nom de "Trouble du Spectre Autistique" (TSA) en supprimant certaines entités nosographiques telles que le syndrome d'Asperger (Annexe 3). 


\section{2. ÉTIOLOGIE}

Malgré de nombreux débats ces dernières années, il est établi à l'heure actuelle que l'autisme est une maladie d'origine multifactorielle, sans étiologie unique retrouvée.

Il existe des facteurs de risques environnementaux :

Infections et/ou complications pré, péri et postnatales, âge paternel élevé, primiparité, mère née à l'étranger $[a, b]$.

Il existe également des facteurs de risques génétiques :

Augmentation du risque chez les garçons, chez les jumeaux monozygotes et avec antécédents familiaux. On retrouve dans la littérature des atteintes de plusieurs gènes : délétion 22q11, SHANK3 (formation des synapses), PTEN (croissance cérébrale), NLGN3, NRXN1, CNTNAP2, GABRB3, CHD2, HDAC4, GDI1, SETD5, MIR137, HDAC9 [c, d, e].

De plus certaines anomalies ont été retrouvées grâce aux techniques d'imagerie cérébrale :

Atteinte des neurones miroirs, déficit de connexions cérébrales, faible niveau de substance grise dans les circuits neuronaux thalamo-striato-limbique, excès de développement cérébral entre 1 et 3 ans, altération de la substance blanche au niveau du lobe temporal (jouant sur le langage et la cognition sociale) [f].

Les études biochimiques ont permis de découvrir d'autres anomalies non spécifiques:

Augmentation de la sérotonine, augmentation de la dopamine, augmentation du GABA, diminution de l'ocytocine [g, h]. 


\section{CONTEXTE ET ENJEUX DE L'ÉTUDE}

Cette étude est née de l'intérêt commun entre l'Education Nationale et la responsable du CADIPA, le docteur Brigitte ASSOULINE, concernant les enfants autistes. L'implication des membres de l'Education Nationale dans la prise en charge de l'autisme est un atout à valoriser dans la région Rhône Alpes.

Les parents comme les enseignants peuvent se retrouver démunis face aux comportements spécifiques de ces enfants atteints d'autisme. En effet ces enfants peuvent montrer de grandes facilités dans certains domaines comme de nombreuses difficultés dans d'autres. Ce paradoxe peut être source d'incompréhensions et d'interprétations erronées de la part du corps enseignant ou des parents. En outre ces différences peuvent être source de souffrance, d'anxiété et de repli chez les enfants atteints d'autisme. Il est fondamental de réussir à les intégrer sur le plan scolaire. Cette intégration nécessite de comprendre comment ils fonctionnent, comment ils apprennent ainsi que les spécificités cognitives qu'ils présentent. Une aide spécifique et des aménagements pédagogiques adaptés pourront ainsi être mis en place.

\section{Auxiliaires de Vie Scolaire (AVS):}

Les Auxiliaires de Vie Scolaire sont chargées du suivi d'un ou plusieurs élèves handicapés. Elles peuvent effectuer diverses activités en fonction des besoins des élèves. Leurs missions principales sont :

- Interventions dans les classes (aide pour manipuler le matériel, faciliter la communication) et en dehors des temps d'enseignement (repas, interclasses, devoirs)

- Participation aux sorties de classe (éviter l'exclusion, aider l'enfant dans les activités)

- Aide concernant des gestes techniques ne requérant pas une qualification médicale ou paramédicale (gestes d'hygiène)

- Collaboration à la mise en œuvre et au suivi des projets de scolarisation

La demande d'AVS doit être faite auprès de la Maison Départementale des Personnes Handicapées (MDPH) dans le cadre du Projet Personnalisé de Scolarisation (PPS). Le dossier est ensuite transmis à la Commission des Droits et de l'Autonomie des Personnes Handicapées (CDAPH) qui attribue et définit des domaines d'activité de l'AVS ainsi qu'un quota d'heures. 


\section{MISE EN PLACE DE L'ÉTUDE}

La première étape de l'étude a duré 8 mois. Nous avons sélectionné les files actives des années 2010 et 2011 du Centre Alpin du DIagnostic Précoce de l'Autisme (CADIPA), grâce à l'aide précieuse du Département d'Information Médicale (DIM) du Centre Hospitalier Alpes Isère (384 patients). Puis un travail long et minutieux de recherche et d'extraction de données a été effectué. Chaque dossier a été lu et examiné dans le but de respecter les critères d'inclusion et d'exclusion que nous avions mis en place (disponibles dans l'article). Parmi les patients inclus (83 patients), une recherche plus approfondie dans les dossiers a permis d'extraire plusieurs variables détaillées ci-dessous.

\section{$>$ Variables quantitatives:}

- Âge

- Nombre d'heures d'Auxiliaire de Vie Scolaire (AVS)

- Indice du WISC-IV : Indice de Compréhension Verbale (ICV), Indice de Raisonnement Perceptif (IRP), Indice de Mémoire de Travail (IMT), Indice de Vitesse de Traitement (IVT)

\section{Variables dichotomiques:}

- Sexe

- Lieu de résidence

- Type de scolarisation

- Prise en charge psychiatrique, psychologique, orthophonique, orthoptique, psychomotrice, ergothérapeutique, kinésithérapique

\section{Variables catégorielles:}

- Diagnostic

- Niveau de comorbidité psychiatrique

- Présence d'une AVS

- Niveau de scolarisation

- Nombre de prises en charge par semaine

- Type de test neuropsychologique

- Temps de scolarisation 
La deuxième étape de l'étude a duré 4 mois. Nous avons recueilli l'ensemble des données brutes puis créé certaines variables catégorielles selon la distribution des données ou de leur intérêt pour la clinique. L'analyse statistique a été effectuée par le Docteur Andrea PIERO à l'aide du logiciel SPSS.

La dernière partie de l'étude a été consacrée à la valorisation et la diffusion des résultats en passant par l'écriture, la mise en page de l'article selon les normes éditoriales de L'Encéphale puis les recherches d'articles similaires afin d'étoffer, d'élaborer et d'approfondir la discussion. Nous avons par la suite soumis notre article à la revue l'Encéphale. 
Après 6 mois d'attente, nous avons reçu une première réponse des réferees, avec un accord partiel sous réserve de modifications, en avril 2014 (Annexe 4). L'article a été retravaillé conformément aux conseils du réferee, envoyé pour relecture, puis validé en juin 2014 pour publication au sein de la revue l'Encéphale (Annexe 5).

Grâce à cet article ainsi qu'aux autres études effectuées au CADIPA, nous avons eu le plaisir d'apprendre que la ville de Grenoble a été choisie au sein de la région Rhône-Alpes pour initier l'expérimentation d'unités d'enseignement spécifiques dans le cadre du troisième plan autisme (2013-2017) [i].

L'inauguration de ces unités est prévue en septembre 2014 grâce au partenariat entre l'Education Nationale et le médico-social. 
La scolarisation des patients atteints par des Troubles du Spectre Autistique sans retard mental

\section{${ }^{1}$ I GRIMM Dévi, ${ }^{2}$ ASSOULINE Brigitte, ${ }^{3}$ PIERO Andrea*}

${ }^{1}$ Interne, Centre Hospitalier Alpes Isère, Saint Egrève 38120; Université Joseph Fourier, Grenoble, 38000

${ }^{2}$ Praticien Hospitalier, Centre Hospitalier Alpes Isère, CADIPA, Centre Expert Asperger, 3 Rue de la Gare, Saint Egrève 38120

${ }^{3}$ I Praticien Hospitalier, Centre Hospitalier Alpes Isère, Comité de Soutien à la Recherche, 3 Rue de la Gare, Saint Egrève 38120 


\section{$\underline{\text { Résumé }}$}

La scolarité des enfants atteints de Troubles du Spectre Autistique Sans Déficit Intellectuel (TSA SDI) est un sujet encore peu exploré en France. L'objectif de cette étude était d'évaluer un certain nombre de facteurs cliniques et cognitifs qui peuvent être liés à la fonction de l'Auxiliaire de Vie Scolaire (AVS). 83 patients autistes (3-18 ans) sans retard mental ont été inclus au Centre Alpin de DIagnostic Précoce de l'Autisme (CADIPA), Centre Ressources Autisme Rhône Alpes. Nos résultats montrent que les autistes typiques et atypiques sont principalement retrouvés en maternelle et au primaire, ils bénéficient plus fréquemment d'une AVS et d'un plus grand nombre de prises en charge comparativement aux enfants porteurs du syndrome d'Asperger. Les profils cognitifs de la Wechsler Intelligence Scale for Children (WISC 4e éd) sont également différents: l'Indice de Compréhension Verbale (ICV) et l'Indice de Mémoire de Travail (IMT) sont plus élevés chez les Asperger que chez les autistes typiques et atypiques. L'ICV et l'Indice de Vitesse de Traitement (IVT) sont indirectement corrélés au nombre d'heures d'AVS. Dans le milieu scolaire, la présence d'AVS semble associée au diagnostic principal et au fonctionnement neuropsychologique selon un continuum qui va de l'autisme typique (besoins et déficits plus importants) au syndrome d'Asperger. Aucun lien entre la comorbidité psychiatrique et la scolarité n'a été retrouvé.

Mots clés : Autisme, Troubles Envahissants du Développement, Retard Mental, Spectre Autistique, Scolarité 


\section{$\underline{\text { Abstract }}$}

The education of children with Autism Spectrum Disorder without mental retardation is a subject still little explored in the literature. Aims: The objective of this study was to evaluate a number of clinical, personal and cognitive factors that may be related to the use of Auxiliary Life School (according to French Education System). Methodology: 83 autistic patients (3-18 years; 73 man and 10 women) without mental retardation and taking schooling were included in the Alpine Centre for Early Diagnosis of Autism. Results: the sample included: 45 subjects with typical autism, 12 subjects with atypical autism and 26 subjects with Asperger. Our results show that typical and atypical autism are mainly found in the nursery and primary school, they have a more frequently Auxiliary Life School and a larger number of consultations and treatment episodes compared with Aspergers. Cognitive profiles obtained with theWechsler Intelligence Scale for Children, 4e edition (WISC-IV) also differ: the Verbal Comprehension Index (VCI) and Working Memory Index (WMI) are higher than Aspergers for the typical and atypical autism. The VCI and Processing Speed Index (PSI) are indirectly correlated to the number of hours of Auxiliary Life School. No link between psychiatric comorbidity, king of psychological and psychiatric treatment and education were found. Conclusion: Using Auxiliary Life School seems to be associated with the autism spectre disorder diagnosis and with neuropsychological functioning as assessed by WISC IV, along a continuum that ranges from typical autism (more needs and deficits) to Asperger syndrome through atypical autism.

Key words : Autism, School, Mental Retardation, Asperger, Education 


\section{Introduction}

Les Troubles du Spectre Autistique (TSA) appartiennent aux Troubles Envahissants du Développement (TED). Ils apparaissent avant l'âge de 3 ans et persistent à l'âge adulte. Ils sont caractérisés par un trouble des interactions sociales, un trouble de la communication verbale et non verbale ainsi que par des comportements stéréotypés et/ou des intérêts restreints [1].

La prévalence des TSA varie de 90 à 120 pour 10000 individus et ne cesse de progresser. Le sexe ratio est de 5 hommes pour 1 femme [2, 3]. Il y a 9/10000 autistes typiques, 15,3/10000 autistes atypiques et 3/10000 syndromes d'Asperger dont 62\% de retard mental [4, 5], mais les écarts de prévalence retrouvés dans les travaux et enquêtes épidémiologiques restent difficiles à interpréter et nous invitent à la prudence [6].

L'accès à la scolarisation de ces enfants autistes ne serait que de $20 \%$ selon le Collectif Autisme [7]. Pourtant les dernières recommandations de la Haute Autorité de Santé (HAS) de mars 2012 mentionnent que tout enfant/adolescent en âge scolaire doit bénéficier d'une scolarisation effective, en milieu ordinaire ou adapté [8]. L'application de la loi du 11 Février 2005 pour l'égalité des droits et des chances des personnes handicapées [9] et de la circulaire du 8 Mars 2005 sur la prise en charge des personnes atteintes d'autisme ou de TED [10] garantissant cet accès à la scolarisation reste donc difficile.

Les études sur les méthodes d'aide à la scolarisation sont fréquentes $[11,12,13]$. Le bénéfice cognitif du temps de scolarité a été démontré dans l'étude de Baghdadli et al. [14]. De plus dans le cadre de leur projet scolaire, l'accompagnement par une AVS est préconisé pour aider les jeunes autistes par le Ministère de l'Education Nationale [15], mais les raisons expliquant le faible taux de scolarité des enfants autistes est un sujet encore peu exploré. Nous avons pu constater dans la littérature [16] un intérêt croissant des chercheurs pour les TSA SDI ou les TED SDI. C'est pour ce groupe de sujets que l'enjeu d'une scolarisation optimale est encore plus important [17].

L'objectif principal de cette étude est d'analyser les liens entre les TSA SDI, les comorbidités psychiatriques et la scolarité. L'objectif secondaire est d'analyser les facteurs cognitifs et contextuels limitant la scolarisation de ces enfants. 


\section{Méthodes}

\section{Échantillon et procédures}

Notre étude rétrospective s'est effectuée au CADIPA, antenne grenobloise du Centre de Ressource Autisme Rhône-Alpes (CRA RA), hébergée par le Centre Hospitalier Alpes Isère (CHAI). Cette étude a été soumise à l'accord du Comité d'Ethique et déclarée à la CNIL (Annexe 6).

Nous avons sélectionné les patients diagnostiqués autistes sur les bases de la CIM-10 (Classification Internationale des Maladies) : F84.0-autisme typique, F84.1-autisme atypique, F84.5-syndrome d'Asperger. Notre étude portant sur les TSA SDI, nous avons exclu tous les patients avec retard mental. Puis nous avons affiné notre échantillon en excluant les patients non scolarisés en milieu ordinaire (hospitalisations, Institut Médico-Educatif, déscolarisation, enfants de moins de 3 ans). Les critères d'inclusion étaient donc : 1) patients porteurs d'un diagnostic d'autisme typique, atypique ou d'un syndrome d'Asperger, 2) sans retard mental, 3) scolarisés au moment de l'extraction des données.

Dans un premier temps, nous avons extrait les files actives anonymes des années 2010 et 2011 du CADIPA. L'échantillon initial était composé de 384 patients dont 193 autistes typiques, atypiques et syndromes d'Asperger. Dans ces 193 patients, on retrouvait 36 patientes de sexe féminin (19\%) et 157 patients de sexe masculin (81\%). 301 patients ont étés exclus pour les raisons suivantes: 104 patients diagnostiqués autres TED et TED sans précision (F84.8 et F84.9 selon la CIM-10), 25 patients non diagnostiqués TED (2 troubles du langage, 17 Troubles d'Hyperactivité et Déficit Attentionnel (THADA), 2 troubles anxieux, 4 épisodes dépressifs majeurs), 38 patients sans diagnostic retrouvé (patients en cours de bilan, perdus de vue), 36 autistes avec retard mental, 23 autistes en IME (6 sans retard mental + 17 avec retard mental), 1 autiste hospitalisé, 74 autistes non scolarisés dont 1 en âge de l'être.

L'échantillon final était composé de 83 patients atteints de TSA SDI (22 en maternelle, 33 en primaire, 24 au collège, 4 au lycée), dont 73 de sexe masculin et 10 de sexe féminin : 45 autistes typiques, 12 autistes atypiques et 26 Aspergers. 40 présentaient des comorbidités psychiatriques, dont 13 troubles du langage, 13 troubles "DYS" (dyspraxie, dyslexie, dysgraphie, dyscalculie, dysorthographie), 11 troubles attentionnels avec ou sans hyperactivité, 10 troubles anxieux, 4 troubles du sommeil, 3 troubles du comportement alimentaire, 3 troubles du comportement, 2 TOC (Troubles Obsessionnels Compulsifs) et 7 "autres" (dysharmonie psychotique, dysharmonie évolutive, trouble des fonctions exécutives). 43 patients ne présentaient aucune comorbidité psychiatrique. 
Le diagnostic de l'autisme reste essentiellement clinique, mais les recommandations de l'HAS 2010 [18] conseillent l'utilisation de l'ADI-R (Autism Diagnostic Interview Revised) [19], de l'ADOS (Autism Diagnostic Observation Schedule) [20, 21], et de la CARS (Childhood Autism Rating Scale). La démarche diagnostic du CADIPA s'effectue par une équipe pluridisciplinaire. Tous les enfants ont bénéficié d'un bilan diagnostique standardisé qui requiert au minimum la passation de l'ADI, de l'ADOS et d'un bilan cognitif.

\section{Matériels}

ADI-R (Autism Diagnostic Interview Revised) : c'est un outil d'entretien semistructuré [19]. Il concerne les enfants de plus de 3 ans ayant un âge développemental d'au moins 18 mois. Chaque item est coté de 0 à 3 en fonction du degré de sévérité.

ADOS (Autism Diagnostic Observation Schedule) : c'est un outil d'observation semistructuré d'une heure [20, 21]. Il se base sur l'observation de la communication, des interactions sociales réciproques, $\mathrm{du} \mathrm{jeu}$, des comportements stéréotypés et des intérêts restreints. L'ADOS comporte quatre modules correspondant à différents niveaux de développement langagier. Les items sont cotés de 0 à 3.

WISC-IV (Wechsler Intelligence Scale for Children, 4e édition) : c'est un outil d'évaluation de l'efficience intellectuelle pour les enfants âgés de 6 à 16 ans [22, 23]. Ce test est effectué par des psychologues expérimentés. Il est composé de quinze subtests regroupés en quatre indices : indice de compréhension verbale (ICV), indice de raisonnement perceptif (IRP), indice de mémoire de travail (IMT), indice de vitesse de traitement (IVT).

\section{Analyses statistiques}

Toutes les données ont été analysées à 1'aide du programme SPSS (Statistical Package Social Sciences) version 11.0. Dans toutes les analyses, le seuil de signification p a été considéré comme significatif lorsqu'il était inférieur à 0.05 .

Les variables étaient de trois types: A) Variables quantitatives, B) Variables dichotomiques (0/1) et C) Variables catégorielles. La liste des variables sera à disposition à la demande du lecteur intéressé. Les analyses des données ont été effectuées en trois étapes :1) Une description de l'échantillon (tableau 1) ;2) Des mesures d'association entre différentes variables qualitatives ont été recherchées avec le Test du Khi2 (voir résultats). Avec le General Linear Model (GLM) et l'Analyse de la Variance ANOVA, nous avons étudié la relation entre l'utilisation des AVS, le type de diagnostic et la présence de comorbidité 
psychiatrique (tableau 2), et la relation entre diagnostic, âge et résultats aux quatre indices du WISC IV (voir résultats). 3) Afin de trouver les facteurs de prédiction d'utilisation des AVS (tableau 3) une régression linéaire a été utilisée avec les quatre indices du WISC utilisés comme variables indépendantes.

\section{Résultats}

\section{Description de l'échantillon}

Dans le tableau 1, nous montrons les caractéristiques personnelles et cliniques des 83 jeunes patients inclus dans l'étude.

\section{Rôle du diagnostic principal}

Une association significative entre le diagnostic (autisme typique, atypique et syndrome d'Asperger) et le niveau de scolarité (maternelle, primaire, collège, lycée) a été détectée dans notre échantillon. Les autistes typiques et atypiques sont principalement retrouvés en maternelle (35\%) et au primaire (44\%), tandis que les syndromes d'Asperger se retrouvent majoritairement $(54 \%)$ au collège ( chi square $=18.23$; d.f $=6 ; \mathrm{p}<.006)$.

Nous n'avons pas établi de liens significatifs (chi square $=0.031 ;$ d.f $=2 ; \mathrm{p}<.985$ ) entre le diagnostic (autiste typique, atypique, syndrome d'Asperger) et le type de scolarisation (milieu ordinaire ou protégé). Une tendance à la significativité (chi square $=12.17 ;$ d.f $=6 ; \mathrm{p}$ $<$.058) concernant l'association du diagnostic et du temps de scolarité a été retrouvée. Nous avons observé que la majorité des TSA SDI sont scolarisés à plein temps, avec un plus fort pourcentage chez les syndromes d'Asperger (92.3\% vs $83.3 \%$ chez les autistes atypiques et $75.6 \%$ chez les autistes typiques).

Une association fortement significative a été trouvée entre le diagnostic et la présence ou non d'une AVS (chi square $=15.61 ;$ d.f $=2 ; p<.000$ ). $80,5 \%$ des autistes typiques, $63.6 \%$ des autistes atypiques et $32 \%$ des syndromes d'Asperger bénéficiaient d'une AVS.

Le diagnostic et le nombre de prises en charge par semaine sont significativement liés (chi square $=27.83 ;$ d.f $=14 ; \mathrm{p}<$.015). Les autistes typiques et atypiques ont majoritairement de 2 à 4 prises en charge par semaine (respectivement 57,8\% et 75,1\%), tandis que les syndromes d'Asperger se situent entre 0 et 2 prises en charge $(84,2 \%)$. Les syndromes d'Asperger ont beaucoup moins (chi square $=21.59$; d.f $=2 ; \mathrm{p}<.000$ ) de suivi orthophonique $(15.4 \%)$ contrairement aux autres formes d'autisme typique et atypique (66.7\% à $71.1 \%)$. 
La comparaison (contrôlée en fonction de l'âge) entre les diagnostics et les indices du WISC-IV indiquent que : 1) l'ICV diffère significativement $(\mathrm{F}=23.11, \mathbf{p}<. \mathbf{0 0 0} ;$ d.f. = 2 ; partial eta squared $=.576)$ en étant plus élevé pour les syndromes d'Asperger $(126.1 \pm 22.1)$ que pour les autistes atypiques $(90.3 \pm 12.9)$ et les autistes typiques $(85.4 \pm 12.8)$. 2) l'IMT diffère significativement $(\mathrm{F}=8.06, \mathbf{p}<. \mathbf{0 0 1}$; d.f. $=2$; partial eta squared $=.357)$ en étant plus élevé pour les syndromes d'Asperger $(100.1 \pm 18.1)$ que pour les autistes atypiques $(90.3 \pm 8.9)$ et les autistes typiques (77.6 \pm 13.3$)$. Les deux autres indices (IRP, IVT) ne différent pas significativement en fonction du diagnostic.

\section{Rôle de la comorbidité}

Nous n'avons pas trouvé d'association significative entre les comorbidités psychiatriques et la scolarité, notamment pour ce qui concerne le temps de scolarisation (chi square $=21.59 ;$ d.f $=2 ; \mathrm{p}<.297$ ), le type de scolarisation, ordinaire ou protégé (chi square $=$ 5.60; d.f $=2 ; \mathrm{p}<.065$ ), la présence d'une AVS (chi square $=2,40 ;$ d.f $=2 ; \mathrm{p}<.301$ ). De même aucune association significative n'a été trouvée entre les comorbidités psychiatriques et le nombre de prises en charge par semaine (chi square $=18,27$; d.f $=14$; $p<.195$ ), ou entre les comorbidités psychiatriques et les différents types de prise en charge (psychologique, orthophonique, orthoptique, psychomotricienne, ergothérapeutique, kinésithérapique).

\section{Facteurs de prédiction d'utilisation des AVS}

Une association significative a été retrouvée entre le diagnostic et le nombre d'heures d'AVS. Cette association a été testée dans le cadre d'une ANOVA (tableau 2) : l'association a été contrôlée pour les comorbidités psychiatriques et l'interaction entre comorbidité psychiatrique et diagnostic. Une corrélation significative a été retrouvée entre le nombre d'heures d'AVS et certains indices du WISC-IV, en particulier l'indice de vitesse de traitement de l'information (IVT) et l'indice de compréhension verbale (ICV). Plus ces indices étaient bas, plus le nombre d'heures d'AVS était élevé (tableau 3).

\section{Discussion}

Le peu de littérature sur les liens entre TSA SDI et scolarisation en France nous a donné l'occasion d'explorer un domaine encore insuffisamment connu et étudié. Les données trouvées dans cette étude sont innovantes et mériteraient d'être approfondies dans d'autres recherches, afin de faciliter la scolarisation des enfants atteints de TSA. Cependant l'absence 
d'étude comparative limite l'étendue de la discussion et rend nos résultats peu généralisables. Les dimensions de l'échantillon final, la fiabilité du diagnostic (CRA) et le fait d'avoir ciblé une population de TSA SDI semblent être les points forts de l'étude.

En considérant les caractéristiques de notre échantillon, on remarque que nos pourcentages correspondent aux données épidémiologiques actuelles $(5 \mathrm{H} / 1 \mathrm{~F}$; [2]), ce qui rend notre étude représentative de la population atteinte d'autisme. En effet, le sexe ratio des patients ayant un TSA avec ou sans déficit intellectuel était de $19 \%$ de sexe féminin et $81 \%$ de sexe masculin (calculé sur les 193 patients atteints par un autisme typique, atypique et syndrome d'Asperger, avant d'appliquer les autres critères d'exclusion). Dans notre échantillon final (TSA SDI) le pourcentage des patientes de sexe féminin diminue (12\%) et celui des patients de sexe masculin augmente (88\%) se rapprochant ainsi des dernières données épidémiologiques sur les TSA SDI (6H/1F; [18]). Parmi les 83 patients de notre échantillon final de TSA SDI, 54\% des sujets présentent un autisme typique, $14 \%$ un autisme atypique et $31 \%$ un syndrome d'Asperger. A notre connaissance, il n'existe pas de données épidémiologiques dans le cas spécifique des TSA SDI, ce qui rend ces derniers pourcentages non comparables.

Selon le Collectif Autisme, 20\% d'autistes avec ou sans déficit intellectuel sont scolarisés [7]. Dans notre étude, nous avons trouvé 92\% de TSA SDI scolarisés, dont la majorité à temps plein. Ces chiffres sont difficilement comparables car les deux populations sont différentes. La discussion de ce taux élevé de scolarisation doit tenir compte d'un biais de sélection (dû aux caractéristiques des patients adressés au CADIPA). D'autant plus que le pourcentage de syndrome d'Asperger, qui s'intègrent et s'adaptent plus facilement que les autres TSA, n'est pas négligeable dans notre échantillon (31\%). Ces données encouragent les efforts d'intégration faits par les écoles ainsi que les prises en charge des équipes thérapeutiques ces dernières années.

Les autistes typiques et atypiques sont principalement scolarisés en maternelle et en primaire tandis que les syndromes d'Asperger sont principalement scolarisés au collège. Ces résultats sont certainement liés au diagnostic plus tardif des syndromes d'Asperger, qui passent inaperçus dans l'enfance (sur-adaptation sociale), puis se désadaptent à la préadolescence. Il semble que des mesures de repérage précoce et de diagnostic spécifique, une prise en charge rapide et intensive basée sur les dernières connaissances scientifiques permettent un pronostic optimiste pour les jeunes atteints de TED [24]. Toutefois, nous pouvons nous poser la question du devenir scolaire de ces enfants atteints d'autisme typique et 
atypique, retrouvés moins fréquemment au collège et au lycée. Cet effet pyramide peut être expliqué par l'orientation fréquente en milieu spécialisé. En outre le biais de sélection du CADIPA peut également jouer un rôle ici. En effet les autistes typiques et atypiques sont adressés et donc diagnostiqués plus tôt que les Aspergers.

De façon attendue, les autistes typiques et atypiques bénéficient en grande majorité d'une AVS contrairement aux Aspergers. Cependant le recours à une AVS, par son accompagnement et la relation instaurée avec les parents, peut participer à une prise en charge globale de l'enfant [25], qui pourrait également être bénéfique aux enfants Aspergers. Il serait intéressant d'évaluer dans des études ultérieures les résultats scolaires obtenus par ces patients atteints de TSA SDI en comparaison à d'autres élèves sans aucun trouble du développement ainsi qu'une comparaison entre les jeunes suivis par une AVS et ceux qui ne le sont pas.

Concernant les comorbidités psychiatriques, étonnamment nous n'avons pas trouvé d'association significative avec les différents indices de scolarité que nous avons extrait (type, niveau et temps de scolarisation, présence et nombre d'heure d'AVS). La présence des comorbidités psychiatriques ne semble pas modifier les orientations et les choix vis-à-vis de la scolarisation de ces sujets. Trois considérations s'imposent : 1) la première est que les indices utilisés pourraient ne pas être suffisamment fins pour détecter des différences statistiquement significatives; 2) la deuxième est que les outils disponibles pour la scolarisation de ces sujets ne soient pas conçus pour être adaptés aux besoins sanitaires des TSA SDI, mais plutôt à leur fonctionnement cognitif et neuropsychologique ; 3) la troisième est que les comorbidités restent encore sous-diagnostiquées [16]. L'étude mériterait d'être répliquée et comparée dans d'autres établissements, sur une population plus importante, ou avec l'utilisation d'autres indices (analyse des résultats scolaires, apport et aide spécifique d'une AVS, difficultés signalées par les enseignants...).

Nous avons retrouvé que certaines fonctions cognitives sont corrélées à la scolarisation des TSA SDI, notamment deux indices du WISC-IV : la vitesse de traitement (IVT) et la compréhension verbale (ICV).

Plusieurs travaux existent dans la littérature sur les profils cognitifs chez les autistes, mais peu utilisent le WISC-IV (2003), pour ce qui est à notre connaissance. Dans les quelques études qui l'utilisent, on note chez les patients autistes, des valeurs plus basses dans les indices de vitesse de traitement (IVT) et de mémoire de travail (IMT) comparativement aux indices de raisonnement perceptif (IRP) et de compréhension verbale (ICV) [26]. Des différences existent entre les Aspergers et les autistes de haut niveau, notamment au niveau 
des habilités visuo-spatiales [27]. L'ICV serait plus élevé chez les syndromes d'Asperger et les autistes non déficitaires [28]. Ce dernier point pourrait expliquer l'adaptation plus importante des TSA SDI dans la population générale et au sein d'un établissement scolaire, le langage étant l'une des bases de la communication et des interactions sociales.

Les résultats obtenus dans notre échantillon semblent confirmer que les syndromes d'Asperger ont des performances cognitives supérieures aux patients atteints par un TSA, même sans déficit intellectuel. En effet, on a pu constater que l'ICV et l'IMT sont plus bas chez les autistes typiques et plus élevés chez les syndromes d'Asperger. Les autistes atypiques s'insèrent entre les deux. Mais nos résultats indiquent aussi que deux indices sur quatre du WISC-IV sont corrélés à une utilisation majeure d'AVS : un ICV plus bas (indice qui semble être fortement lié au diagnostic), et un IVT plus bas (qui ne semble pas dépendre du diagnostic). En effet ces deux indices sont indirectement corrélés au nombre d'heures d'AVS dans notre étude.

Même si ces indices ont probablement été calculés après la mise en place des AVS, il semble évident que ces sujets aient été détectés sur le plan clinique et socioéducatif comme porteurs de besoins plus importants. Il pourrait être judicieux d'utiliser de façon plus systématique ces indices pour évaluer le nombre d'heures d'AVS nécessaires ainsi que pour cibler les manques et les besoins spécifiques des enfants scolarisés ayant un TSA SDI. Des interventions plus ciblées des AVS en fonction des diagnostics et des difficultés propres des patients, ainsi que des formations spécifiques au sein des écoles pourraient être proposées. Dans ce but, nos résultats nécessitent d'être confirmés par d'autres investigations.

L'objectif de cette étude pilote était aussi de sensibiliser les chercheurs à ce sujet : les résultats semblent souligner que dans un milieu scolaire ordinaire, l'utilisation de l'AVS est associée au diagnostic et au fonctionnement neuropsychologique, selon un continuum qui va de l'autisme typique (besoins plus importants, déficits plus significatifs) au syndrome d'Asperger (besoins moins importants, déficits moins significatifs) en passant par l'autisme atypique qui s'insère entre les deux. Les comorbidités psychiatriques et les prises en charge de celles-ci semblent peu influencer la scolarisation des ces patients.

D'autres études à plus grande échelle permettraient d'approfondir ces questions passionnantes sur les enfants autistes sans retard mental afin de les aider et de les accompagner au mieux tout au long de leur scolarité.

\section{Conflit d'intérêt : aucun.}




\section{$\underline{\text { Tableaux }}$}

Tableau 1. Description de l'échantillon.

\begin{tabular}{|c|c|c|}
\hline Variables & Moyenne & Range \\
\hline Age & $9.2 \pm 3.9$ & Range 3-18 \\
\hline WIVT & $82.6 \pm 15.8$ & Range 50-118 \\
\hline WICV & $104.1 \pm 26.4$ & Range 53-179 \\
\hline WIRP & $95.4 \pm 19.4$ & Range 51-130 \\
\hline WIMT & $82.6 \pm 15.7$ & Range 50-118 \\
\hline Heures Auxiliaire Vie Scolaire & $6.7 \pm 6.1$ & Range $0-24$ \\
\hline Variables & & Pourcentage $(\%)$ \\
\hline \multirow[t]{2}{*}{ Sexe } & Masculin & $73(88 \%)$ \\
\hline & Féminin & $10(12 \%)$ \\
\hline \multirow[t]{2}{*}{ Résidence } & Isère & $64(77 \%)$ \\
\hline & Haute Savoie & $19(23 \%)$ \\
\hline \multirow[t]{3}{*}{ Diagnostic } & Autisme typique (F84.0) & $45(54 \%)$ \\
\hline & Autisme atypique (F84.1) & $12(14 \%)$ \\
\hline & Asperger $(\mathrm{F} 84.5)$ & $26(31 \%)$ \\
\hline \multirow[t]{3}{*}{ Comorbidité psychiatrique } & Pas de comorbidité & $43(52 \%)$ \\
\hline & Une comorbidité & $20(24 \%)$ \\
\hline & Deux ou plus comorbidités & $20(24 \%)$ \\
\hline \multirow[t]{4}{*}{ Niveau de scolarisation } & Maternelle & $22(26 \%)$ \\
\hline & Primaire & $34(41 \%)$ \\
\hline & Collège & $23(28 \%)$ \\
\hline & Lycée & $4(5 \%)$ \\
\hline \multirow[t]{2}{*}{ Type de scolarisation } & Milieu ordinaire & $76(91 \%)$ \\
\hline & Milieu protégé & $7(9 \%)$ \\
\hline \multirow[t]{5}{*}{ Temps de scolarisation } & Plein temps & $68(82 \%)$ \\
\hline & Entre un plein temps & \\
\hline & et un mi-temps & $2(2 \%)$ \\
\hline & Mi-temps & $10(12 \%)$ \\
\hline & Moins d'un mi-temps & $3(4 \%)$ \\
\hline Présence d'une Auxiliaire de & Non & $30(36 \%)$ \\
\hline \multirow[t]{2}{*}{ Vie Scolaire } & Oui & $49(59 \%)$ \\
\hline & Non connu & $4(5 \%)$ \\
\hline \multirow[t]{3}{*}{ Bilan neuropsychologique } & WISC & $38(46 \%)$ \\
\hline & Autres & $22(27 \%)$ \\
\hline & Absence de bilan & $23(28 \%)$ \\
\hline \multirow{8}{*}{$\begin{array}{l}\text { Nombre de prise en charge } \\
\text { par semaine }\end{array}$} & 0 & $12(14 \%)$ \\
\hline & 1 & $19(23 \%)$ \\
\hline & 2 & $19(23 \%)$ \\
\hline & 3 & $14(17 \%)$ \\
\hline & 4 & $14(17 \%)$ \\
\hline & 5 & $2(2 \%)$ \\
\hline & 6 & $2(2 \%)$ \\
\hline & 7 & $1(1 \%)$ \\
\hline \multirow[t]{2}{*}{ Prise en charge psychiatrique } & Non & $65(78 \%)$ \\
\hline & Oui & $18(22 \%)$ \\
\hline Prise en charge psychologique & Non & $30(36 \%)$ \\
\hline
\end{tabular}




\begin{tabular}{|ccc|}
\hline & Oui & $53(64 \%)$ \\
Prise en charge orthoptique & Non & $75(90 \%)$ \\
& Oui & $8(10 \%)$ \\
Prise en charge orthophonique & Non & $39(47 \%)$ \\
Prise en charge psychomotrice & Oui & $44(53 \%)$ \\
& Non & $63(76 \%)$ \\
Prise en charge ergo- & Oui & $20(24 \%)$ \\
thérapeutique & Non & $67(81 \%)$ \\
Prise en charge kinési- & Oui & $16(19 \%)$ \\
thérapeutique & Non & $81(97 \%)$ \\
\hline
\end{tabular}

Note. WISC, Wechsler Intelligence Scale for Children.CMP = Centre Médicaux-

Psychologique $; \mathbf{H J}=$ Hôpital de jour ; CATTP = Centre d'Accueil Thérapeutique à Temps Partiel.

Tableau 2. Utilisation des Auxiliaires de Vie Scolaire : interaction entre diagnostic et comorbidité psychiatrique.

\begin{tabular}{cccccccccc} 
Autisme & Autisme & Asperger & \multicolumn{2}{c}{ F1 } & \multicolumn{2}{c}{ F2 } & \multicolumn{2}{c}{ F3 } \\
Typique & Atypique & & F & P & F & P & F & P \\
A (42) & B (11) & C (26) & & & & & &
\end{tabular}

\begin{tabular}{llllllllll}
\hline Heures d'AVS & $8.66 \pm 5.53$ & $6.72 \pm 5.62$ & $3.61 \pm 6.11$ & $\mathbf{3 . 6 7}$ & $\mathbf{. 0 3 0}$ & 1.81 & .170 & .792 & .534
\end{tabular}

Factor 1 F1 $=$ diagnostic; $\underline{\text { Factor } 2 \text { F2 }}=$ comorbidité psychiatrique; Factor 3 F3 $=$ diagnostic $*$ comorbidité psychiatrique. Note Diagnostic $=$ Autisme, Autisme atypique, Asperger . Comorbidité psychiatrique : $0=$ aucune, $1=$ un trouble, $2=2$ ou plus troubles.

Tableau 3. Facteurs de prédiction d'utilisation des Auxiliaires de vie scolaire: régression linéaire multiple (stepwise forward).

\begin{tabular}{ccccccc}
\hline Model & R square & Variables & B & $\begin{array}{c}\text { Standard } \\
\text { Error }\end{array}$ & T & Sig \\
\multirow{2}{*}{1} & \multirow{2}{*}{.222} & Costant & 23.08 & 5.604 & 4.11 &, 000 \\
& & WIVT & -.198 & .067 & -2.97 &, 006 \\
2 & \multirow{2}{*}{.327} & Costant & 30.41 & 6.295 & 4.83 &, 000 \\
& & WIVT & -.183 & .063 & -2.89 &, 007 \\
& & WICV & .081 & .037 & 2.15 &, 039 \\
\hline
\end{tabular}

Model 1 Prédicteurs: (Constant), WIVT ; Model 2 Prédicteurs: (Constant), WIVT, WICV.

Note. Autres variables incluses dans l'analyse: âge, WIRP, WIMT; WISC, Wechsler Intelligence Scale for Children. WIVT (WISC) : Indice de Vitesse de Traitement ; WICV (WISC) : Indice de Compréhension Verbale; WIRP(WISC) : Indice de Raisonnement Perceptif; WIMT (WISC) : Indice de Mémoire de Travail. 


\section{CONCLUSION}

La scolarisation des patients atteints par des Troubles du Spectre Autistique

sans retard mental

\section{Thèse soutenue par Dévi GRIMM}

L'autisme est un trouble neurodéveloppemental d'origine multifactorielle. Il résulte d'interactions complexes entre des facteurs de vulnérabilité génétique et des facteurs environnementaux. L'autisme est caractérisé par des altérations dans le domaine des interactions sociales et de la communication verbale et non verbale ainsi que par la présence d'intérêts et d'activités restreints et stéréotypés. Les symptômes apparaissent durant les trois premières années de la vie.

L'enjeu d'une scolarisation pour ces enfants atteints d'autisme est primordial dans leurs prises en charge, notamment pour les enfants ne souffrant pas de déficit intellectuel.

À partir des 384 patients extraits des files actives des années 2010 et 2011 du Centre Alpin du DIagnostic Précoce de l'Autisme (CADIPA), 83 jeunes autistes étaient éligibles. Parmi eux nous avons retrouvé 92\% de Trouble du Spectre Autistique Sans Déficit Intellectuel (TSA SDI) scolarisé, dont la majorité à temps plein. Les autistes typiques et atypiques étaient principalement scolarisés en maternelle et en primaire tandis que les syndromes d'Asperger étaient retrouvés au collège. Le nombre d'heures d'Auxiliaire de Vie Scolaire (AVS) était plus conséquent chez les autistes typiques et atypiques contrairement aux Aspergers. Aucune association significative n'a été retrouvée entre les comorbidités psychiatriques et les différents indices de scolarité (type, niveau et temps de scolarisation, présence et nombre d'heure d'AVS).

Concernant les capacités cognitives, étudiées à l'aide du WISC-IV (Wechsler Intelligence Scale for Children $4 e$ edition), nos résultats montrent de meilleures performances chez les Aspergers que chez les autistes typiques et atypiques sans déficit intellectuel. En effet les Aspergers ont de meilleurs résultats au niveau de l'Indice de Compréhension Verbale (ICV) et de l'Indice de Mémoire de Travail (IMT).

Notre étude montre également une corrélation entre certains indices du WISC-IV et l'utilisation d'AVS. Plus l'Indice de Vitesse de Traitement (IVT) et l'Indice de Compréhension Verbale (ICV) sont bas, plus le nombre d'heure d'AVS augmente, ce qui pourrait permettre d'utiliser ces indices pour cibler les déficits et besoins en AVS des jeunes autistes.

L'utilisation d'AVS semble associée au diagnostic et au fonctionnement neuropsychologique, selon un continuum qui va de l'autisme typique au syndrome d'Asperger, l'autisme atypique s'insérant entre les deux.

VU ET PERMIS D'IMPRIMER

\section{LE DOYEN}

J.P. ROMANET
Grenoble, le $15 / \mathrm{t} / 14$

LE PRESIDENT DE LA THESE

Professeur BOUGEROL 
[a] Chaste P, Leboyer M. Autism risk factors: genes, environment, and gene-environment interactions. Dialogues Clin Neurosci 2012; 14(3): 281-92

[b] Noterdaeme M. Autism spectrum disorders-an overview of the current research status. Klin Padiatr 2011; 223 Suppl 1: E1-E15

[c] Pinto D, Delaby E, Merico D et al. Convergence of genes and cellular pathways dysregulated in autism spectrum disorders. Am J Hum Genet 2014; 94(5): 677-94

[d] Abrahams BS, Geschwind DH. Advances in autism genetics: on the threshold of a new neurobiology. Nature Reviews Genetics 2008; 9(5): 341-355

[e] SiniscalcolD, Cirillo A, Bradstreet JJ et al. Epigenetic findings in autism : new perspectives for therapy. Int J Environ Res Public Health 2013; 10(9): 4261-73

[f] Lauvin MA, Martineau J, Destrieux C et al. Functional morphological imaging of autism spectrum disorders: current position and theories proposed. Diagn Interv Imaging 2012; 93(3): 139-47

[g] Quaak I, Brouns MR, Van de Bor M. The dynamics of autism spectrum disorders : how neurotoxic compounds and neurotransmitters interact. Int J Environ Res Public Health 2013; 10(8): 3384-408

[h] Gordon I, Vander Wyk BC, Bennett RH et al. Oxytocin enhances brain function in children with autism. Proc Natl Acad Sci U S A 2013; 110(52): 20953-8

[i] http://www.social-sante.gouv.fr/actualite-presse,42/breves, 2325/presentation-du-3emeplan-autisme, $15797 . h t m l$

[1] American Psychiatric Association. Diagnostic and Statistical Manual of Mental Disorders (4 ${ }^{\text {th }}$ Edition). American Psychiatric Association, Washington DC, USA; 2000 [Text revision (DSM-IV-TR)]

[2] Fombonne E, Elsabbagh M, Clarke ME, et al. Épidémiologie de l'autisme. Encyclopédie sur le développement des jeunes enfants 2012; 1-5. http://www.enfantencyclopedie.com/documents/FombonneFRxp1.pdf

[3] King M, \&Bearman P. Diagnostic change and the increase in prevalence of autism. International Journal of Epidemiology 2009; 38(5): 1224-1234

[4] Bailly D, Bouvard M, Casadebaig F et al. Troubles mentaux : dépistage et prévention chez l'enfant et l'adolescent. INSERM dossier de presse [Internet] 2003; 1-9. www.inserm.fr

[5] Autism and Developmental Disabilities Monitoring Network Surveillance Year 2008 Principal Investigators. Prevalence of autism spectrum disorders--Autism and Developmental Disabilities Monitoring Network, 14 sites, United States, 2008. MMWR Surveill Summ 2012; 61(3):1-19

[6] Lenoir P, Bodier C, Desombre H, et al.Sur la prévalence de l'autisme et des troubles envahissants du développement (TED). Encephale 2009; 35(1): 36-42

[7] Collectif Autisme. La scolarisation : apprendre à vivre et grandir parmi les autres. http://www.collectif-autisme.org/scolarisation.html

[8] HAS et Anesm. Autisme et autres troubles envahissants du développement : interventions éducatives et thérapeutiques coordonnées chez l'enfant et l'adolescent. Service Bonnes pratiques professionnelles (HAS) et service Recommandations (Anesm); mars 2012

[9] Loi $\mathrm{n}^{\circ}$ 2005-102 du 11 février 2005pour l'égalité des droits et des chances, la participation et la citoyenneté des personnes handicapées, JORF, 12 février 2005, n³6, page 2353

[10] Circulaire interministérielle $\mathrm{n}^{\circ} 2005-124$ du 8 mars 2005 relative à la politique de prise en charge des personnes atteintes d'autisme et de troubles envahissants du développement (TED), BO, Bulletin Officiel de l'Education Nationale, 14 avril 2005, n ${ }^{\circ}$ 15, p I-XXVII 
[11] Jung S , Sainato DM. Teaching play skills to young children with autism. J Intelligence Dev handicap 2013; 38(1): 74-90

[12] Mason RA, Rispoli M, Ganz JB, et al. Effects of video modeling on communicative social skills of college students with Asperger syndrome. Dev Neurorehabil. 2012; 15(6): 425-34

[13] Reichow B, Barton EE, Boyd BA, et al. Early intensive behavioral intervention (EIBI) for young children with autism spectrum disorders (ASD). Cochrane Database Syst Rev 2012; 10: CD009260

[14] Baghdadli A, Assouline B, Sonié S, et al. Developmental Trajectories of Adaptive Behaviors from Early Childhood to Adolescence in a Cohort of 152 Children with Autism Spectrum Disorders. J Autism Dev Disord. 2012; 4:1314-1325

[15] Scolariser les élèves autistes ou présentant des troubles envahissants du développement. 2009. Collection Repère Handicap, Ministère de l'Eduction nationale, Direction générale de l'Enseignement scolaire, CNDP.

[16] Soussana M, Sunyer B, Pry R, Baghdadli A. Troubles anxieux dans les troubles envahissants du développement sans retard mental : état des lieux chez les enfants et les adolescents au travers d'une revue de la littérature. Encephale 2012; 38(1): 16-24

[17] Chung YC, Carter EW, Sisco LG. Social interactions of students with disabilities who use augmentative and alternative communication in inclusive classrooms. Am $J$ Intelligence Dev handicap 2012; 117(5): 349-67

[18] HAS. Autisme et autres troubles envahissants du développement, État des connaissances hors mécanismes physiopathologiques, psychopathologiques et recherche fondamentale. Service des bonnes pratiques professionnelles; janvier 2010

[19] Lord, C, Rutter, M, et Le Couteur, A. Autism Diagnostic Interview Revised : A revised version of a diagnotic interview for caregivers of individualswith possible pervasive developmental disorders. J Autism Dev Disord 1994; 24(5): 659-685

[20] Lord C, Rutter M, Goode S et al. Autism diagnostic observation schedule: a standardized observation of communicative and social behavior. J Autism Dev Disord 1989; 19(2): 185212

[21] Lord C, Rutter M, Risi S, et al. .Autism Diagnostic Observation Schedule-Generic : A standard measure of social and communication deficits associated with the spectrum of autism. J Autism Dev Disord 2000; 30(3): 205-223

[22] Wechsler D. Wechsler Intelligence Scale for Children-Fourth Edition (WISC-IV). New York : Psychological corporation (2003)

[23] Sattler JM, Dumont R. Assessment of children : WISC-IV and WPPSI-III supplement. La mesa, CA: Jerome Sattler (2004)

[24] Kochman F, Bach E, Dereux A, et al. Le devenir des troubles envahissants du développement après l'adolescence. Encephale 2010; 36(3): 54-57

[25] Krieger AE, Saïas T, Adrien JL. Promouvoir le partenariat parents-professionnels dans la prise en charge des enfants atteints d'autisme. Encephale, 2013; 39(2): 130-136

[26] Oliveras-Rentas RE, Kenworthy L, Roberson RB 3e, et al. WISC-IV profile in highfunctioning autism spectrum disorders: impaired processing speed is associated with increased autism communication symptoms and decreased adaptive communication abilities. J Autism Dev Disord. 2012, 42(5): 655-64

[27] Sahyoun CP, Soulières I, Belliveau JW, et al. Cognitive differences in pictorial reasoning between high-functioning autism and Asperger's syndrome. J Autism Dev Disord. 2009; 39(7): 1014-23

[28] Girardot AM, De Martino S, C Chatel, et al. Profils cognitifs dans les troubles envahissants du développement. Encephale, 2012; 38(6): 488-95 
8. EXEMPLE D'ITEM DE L'ADI-R. 
Critères diagnostiques du $\mathbf{F 8 4 . 0}[299.00]$ Trouble autistique

A. Un total de six (ou plus) parmi les éléments décrits en (1), (2) et (3), dont au moins deux de (1), un de (2) ef un de (3)

(1) Altération qualitative des interactions sociales, comme en témoignent au moins deux des éléments suivants:

(a) altération marquée dans l'utilisation, pour réguler les interactions sociales, de comportements non verbaux multiples, tels que le contact oculaire, la mimique faciale, les postures corporelles, les gestes

(b) incapacité à établir des relations avec les pairs, correspondant au niveau du développement

(c) le sujet ne cherche pas spontanément à partager ses plaisirs, ses intérêts ou ses réussites avec d'autres personnes (p. ex. : il ne cherche pas à montrer, à désigner du doigt ou à apporter les objets qui l'intéressent)

(d) manque de réciprocité sociale ou émotionnelle

(2) Altération qualitative de la communication, comme en témoigne au moins un des éléments suivants:

(a) retard ou absence totale de développement du langage parlé (sans tentative de compensation par d'autres modes de communication, comme le geste ou la mimique)

(b) chez les sujets maitrisant suffisamment le langage, incapacité marquée à engager ou à soutenir une conversation avec autrui

(c) usage stéréotypé et répétitif du langage ou langage idiosyncrasique

(d) absence d'un jeu de faire semblant varié et spontané, ou d'un jeu dimitation sociale correspondant au niveau de développement

(3) Caractère restreint, répétitif et stéréotypé des comportements, des intérêts et des activités, comme en témoigne au moins un des éléments suivants:

(a) préoccupation circonscrite à un ou plusieurs centres dintérêts stéréotypés et restreints, anormale soit dans son intensité, soit dans son orientation

(b) adhésion apparemment inflexible à des habitudes ou à des rituels spécifiques et non fonctionnels

(c) maniérismes moteurs stéréotypés et répétitiffs (p. ex. : battements ou torsions des mains ou des doigts,

mouvements complexes de tout le corps)

(d) préoccupations persistantes pour certaines parties des objets 
Critères diagnostiques du F84.4 (299.80) Syndrome d'Asperger

A. Altération qualitative des interactions sociales manifestées dans au moins deux des domaines suivants:

1) Altération marquée dans l'utilisation, pour réguler les interactions sociales, de comportements non verbaux multiples, tels que le contact oculaire, la mimique faciale, les postures corporelles, les gestes

2) Incapacité à établir des relations avec les pairs, appropriées au niveau de développement

3) Le sujet ne cherche pas spontanément à partager ses plaisirs ou ses réussites avec les autres (p. ex.: ne montre pas ce qu'elle fait, n'apporte pas, ne pointe pas l'objet d'intérêt|

4) Manque de réciprocité sociale ou émotionnelle

B. Caractère restreint, répétilif et stéréotypé des comportements, des intérêts et des activités, comme en témoigne au moins un des éléments suivants:

1) Préoccupation circonscrite à un ou plusieurs centres dintérêt stéréotypés et restreints, anormale soit dans lintensité soit dans la nature

2) Adhésion apparemment inflexible à des habitudes ou à des rituels spécifiques et non fonctionnels

3) Maniérismes moteurs stéréotypés et répétitiff (p. ex.: battements ou torsions des mains ou des doigts, mouvements complexes de tout le corps)

4) Préoccupations persistantes pour certaines parties des objets

C. Ce trouble entraîne une altération cliniquement significative du fonctionnement social, professionnel ou dans d'autres domaines importants

D. Il n'existe pas de retard général cliniquement significatif au plan du langage (p. ex. : la personne utilise des mots isolés vers l'âge de 2 ans et des phrases à valeur de communication vers l'âge de 3 ans)

E. Durant la période de l'enfance, il n'y a pas eu de retard significatif sur le plan clinique dans le développement cognitif ni dans le développement, en fonction de l'âge, des capacités d'autonomie, du comportement adaptatif (saú dans le domaine de linteraction sociale) et de la curiosité pour l'environnement

F. Le trouble ne peut s'expliquer mieux par un autre trouble envahissant du développement spécifique ou la schizophrénie 
F84-0 Autisme infantile

Trouble envahissant du développement caractérisé par : a) un développement anormal ou altéré, manifeste avant l'âge de trois ans, avec b) une perturbation caractéristique du fonctionnement dans chacun des trois domaines psychopathologiques suivants : interactions sociales réciproques, communication, comportement (au caractère restreint, stéréotypé et répétitif)- Par ailleurs, le trouble s'accompagne souvent de nombreuses autres manifestations non spécifiques, par exemple des phobies, des perturbations du sommeil et de l'alimentation, des crises de colère et des gestes auto-agressifs-

\section{F84-1 Autisme atypique}

Trouble envahissant du développement, qui diffère de l'autisme infantile par l'âge de survenue ou parce qu'il ne répond pas à l'ensemble des trois groupes de critères diagnostiques d'un autisme infantile. Cette catégorie doit être utilisée pour classer un développement anormal ou altéré, se manifestant après l'âge de 3 ans et ne présentant pas des manifestations pathologiques suffisantes dans un ou deux des trois domaines psychopathologiques nécessaires pour le diagnostic d'autisme (interactions sociales réciproques, communication, comportement restreint, stéréotypé et répétitif) ; il existe toutefois des anomalies caractéristiques dans l'un ou l'autre de ces domaines. L'autisme atypique survient le plus souvent chez les enfants ayant un retard mental profond et un trouble spécifique sévère de l'acquisition du langage, de type réceptif.

\section{F84-5 Syndrome d'Asperger}

Trouble de validité nosologique incertaine, caractérisé par une altération qualitative des interactions sociales réciproques, semblable à celle observée dans l'autisme, associée à un répertoire d'intérêts et d'activités restreint stéréotypé et répétitif- \| se différencie de l'autisme essentiellement par le fait qu'il ne s'accompagne pas d'un déficit ou trouble du langage, ou du développement cognitif- 
Autism Spectrum Disorder

Must meet criteria $A, B, C$, and D:

A. Persistent deficits in social communication and social interaction across contexts, not accounted fot by general developmental delays, and manifest by all 3 of the following:

1. Deficits in social-emotional reciprocity, ranging from abnormal social approach and failure of normal back and forth conversation through reduced sharing of interests, emotions, and affect and response to total lack of initiation of social interaction,

2. Deficits in nonverbal communicative behaviors used for social interaction, ranging from poorly integratedverbal and nonverbal communication, through abnormalities in eye contact and body-language, or deficits in understanding and use of nonverbal communication, to total lack of facial expression or gestures.

3. Deficits in developing and maintaining relationships, appropriate to developmental level (beyond those with caregivers); ranging from difficulties adjusting behavior to suit different social contexts through difficulties in sharing imaginative play and in making friends to an apparent absence of interest in people

B. Restricted, repetitive patterns of behavior, interests, or activities as manifested by at least two of the following:

1. Stereotyped or repetitive speech, motor movements, or use of objects; (such as simple motor stereotypies, echolalia, repetitive use of objects, or idiosyncratic phrases).

2. Excessive adherence to routines, ritualized patterns of verbal or nonverbal behavior, or excessive resistance to change: (such as motoric nituals, insistence on same route or food, repetitve questioning or extreme distress at small changes).

3. Highly restricted, fixated interests that are abnormal in intensity or focus; (such as strong attachment to of preoccupation with unusual objects, excessively circumscribed or perseverative interests).

4. Hyper-or hypo-reactivity to sensory input or unusual interest in sensory aspects of environment; (such as apparent indifference to pain/heat/cold, adverse response to specific sounds or textures, excessive smelling or touching of objects, fascination with lights or spinning objects).

C. Symptoms must be present in early childhood (but may not become fully manifest until social demands exceed limited capacities)

D. Symptoms together limit and impair everyday functioning. 


\begin{tabular}{|c|c|c|}
\hline Severity Level for ASD & Social Communication & $\begin{array}{l}\text { Restricted interests \& repetitive } \\
\text { behaviors }\end{array}$ \\
\hline $\begin{array}{l}\text { Level } 3 \\
\text { 'Requiring very substantial } \\
\text { support' }\end{array}$ & $\begin{array}{l}\text { Severe deficts in verbal and nonverbal } \\
\text { social communication skills cause } \\
\text { severe impairments in functioning; very } \\
\text { limited intiation of social interactions } \\
\text { and minimal response to social } \\
\text { overtures from others. }\end{array}$ & $\begin{array}{l}\text { Preoccupations, focated rtuals and/or } \\
\text { repetitive behaviors markedly interfere } \\
\text { with functioning in all spheres. Marked } \\
\text { distress when rtuals or routnes are } \\
\text { interrupted; very difficut to redirect } \\
\text { from foxated interest or returns to it } \\
\text { quiekly. }\end{array}$ \\
\hline $\begin{array}{l}\text { Level } 2 \\
\text { 'Requiring substantial support' }\end{array}$ & $\begin{array}{l}\text { Marked deficts in verbal and nonverbal } \\
\text { social communication skills; social } \\
\text { imparments apparent even with } \\
\text { supports in place, imted intiation of } \\
\text { social interactions and reduced or } \\
\text { abnormal response to social overtures } \\
\text { from others. }\end{array}$ & $\begin{array}{l}\text { RRBs and/or preoceupations or foxated } \\
\text { interests appear frequently enough to } \\
\text { be obvious to the casual observer and } \\
\text { interfere with functioning in a variety of } \\
\text { contexts. Distress or frustration is } \\
\text { apparent when RRB's are interrupted; } \\
\text { difficult to redrect from fixated intarest. }\end{array}$ \\
\hline $\begin{array}{l}\text { Level } 1 \\
\text { 'Requiring support' }\end{array}$ & $\begin{array}{l}\text { Wethout supports in place, deficits in } \\
\text { social communication cause roticeable } \\
\text { imparments. Has difficulty intiating } \\
\text { social interrctions and demonstrates } \\
\text { clear examples of atypical or } \\
\text { unsuccessiful responses to social } \\
\text { overtures of others. May appear to } \\
\text { have decteased interest in social } \\
\text { interactions. }\end{array}$ & $\begin{array}{l}\text { Rruals and repeitive behaviors (RRB's) } \\
\text { cause significant interference with } \\
\text { functioning in one or more contexts. } \\
\text { Resists attempts by others to interrupt } \\
\text { RRB's or to be redrected from foxated } \\
\text { interest. }\end{array}$ \\
\hline
\end{tabular}


L'ENCEPHALE

revule

depsychiatrie clinique, biologique et thérapeutique

\author{
Rédacteurs en cher : \\ Professeur H. Loo \\ e-mail : h.loosch-sainte-anne.fr \\ Prolesseur J.P Olié \\ e-mail : jp.oliebch-sainte-anne.fr \\ 7, nov Cabanis - 75674 Paris codex 14 \\ Cocroonnateur Editorial: \\ Annie Bloch \\ e-mail : annio_blochbhotmail.fr \\ 22 , avenue de la Ecurdonnais - 75007 Paris
}

$\mathrm{HL} / \mathrm{g}$

Article 2013/103

$$
\begin{gathered}
\text { C.H. SAINT-EGREVE } \\
\text { Reçu le } \\
31 \text { MARS } 2014 \\
\text { Socrétariat } \\
\text { Présidence C.M.E }
\end{gathered}
$$

Mme le Docteur Andrea PIERO

Centre Hospitalier Alpes Isère

Comité de Soutien à la Recherche 3, rue de laGare

38120 SAINT EGREVE

Paris,

Le 27 Mars 2014

Madame et chère Collègue,

Votre article aLa scolarisation des patients atteints par des Troubles du Spectre Autistique sans retard mentab a été accepté par les réferees.

Le premier réferee accepte votre arricle et ne demande pas de modifications. Le second réferee accepte votse article sous réserve de modifications ( $c \mathfrak{c}$ : copic jointe).

La thématique de votre travail renvoie à des publications qui ont déjà été éditées dans l'Encéphale. Il convient que vous les citiez, cela sera pris en compte dans Pévaluation finale de votre travail.

Lapport de modifications ne doit en aucun cas augmenter le volume de l'article qui ne doit impérativement pas dépasser 15 pages tout compris.

Etes-vous d'accord pour apporter ces corrections ou souhaitez-yous les argumenter?

Dans lattente de votre réponse, je vous prie de croire, Madame et chère Collègue, en mes sentiments bien dévoués.

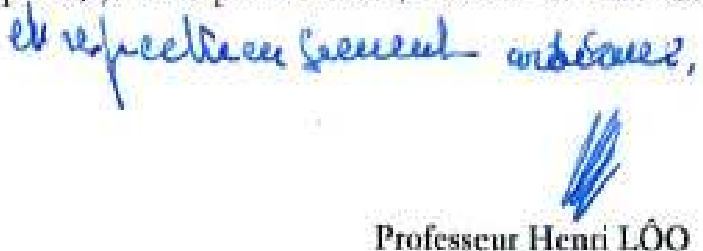


TITRE DE L'ARTICLE : « La scolarisation des paticnts atteints par des Troubles du Spectre Autistique sans retard mentab.

Article 2013/103

\section{Appréciation}

\section{du rapporteur :}

Le travail :

1- est très original

2- contient des faits nouveaux ou

confirme des données incertaines

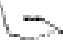

3- ne fait que confirmer des données déjà établies

4- n’a pas de justification expérimentale suffisante

\section{CONTROLES PARTICULIERS :}

Titre $\sqrt{\text { Adéquat }}$ - Inadéquat

Résumés: Adéquats - Inadéquats

Techniques et contróles :

Appropriés Inappropriés

Bibliographie :Adéquate- Inadéquate

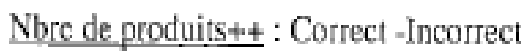

\section{Proposition du rapporteur :}

L'article est à :

a) accepter sans modification ou avec des modifitations rxinimes

b) accepter sous réserve de modifications

c) reconsiderer après modifications importantes

d) refuser

+ Veuillez barrer le terme incorrect de l'alternative,

++ Doivent être utilistés (lorsqu'elles existent) les dénominations communes internationales (DCD) ou les noms chimiques. Les noms déposés peuvent être utilisés exceptionnellement à la condition qu'ils soient mentionnés chaque fois entre parenthèses (N.D).

Veuillez justifier votre position et suggérer les modifications souhaitées (les commentaires doivent être dactylographies).

Veuillez utiliser le verso et éventuellement une feuille blanche.

A retourner : Professew Henvi LOO-Centre Haspitaller Sainte-Anne-Service S.H.U.108, rue de la Santé - 75674 PARIS Cédex IA 
Je viens de réceptionner votre demande de correction de l'article * La scolarisation des patients atteints par des troubles du Spectre Autistique sans retard B, en effet le courrier hospitalier n'est pas toujours acheminé dans les temps. Pour ces raisons je m'excuse de l'absence de réponse et vous communique mes impressions sur cet article.

Cet article contient des faits nouveaux sur la scolarisation des personnes TSA sans déficit intellectuel dans la mesure où il fait le lien entre certaines difficultés cognitives et le besoin d' AVS.

Cette étude confirme également un constat clinique des différentes régions à savoir que les Asperger ont plus de ressources cognitives verbales pour s'adapter a la scolarisation mais qu'lis peuvent avoir besoin d'AVS mais pas seulement ....par exemple l'étude confirme que les Asperger ont des compétences cognitives verbales supérieures aux autres personnes autistes et qu'elles ont moins de prises en charge notamment orthophoniques or la communication est une difficulté centrale dans leurs profils, eux qui sont en difficuité au moment de l'adolescence période des codes langagiers, des phénomènes de groupe et du collège, c'est souvent à cette période que les personnes Asperger décrochent de la scolarisation et écourtent leurs études, d'où une crute de la fréquentation au lycée et dans les ètudes supérieures, comme le montre les résultats de cette étude.

Les auteurs pourraient discuter plus avant sur les besoins différents, en AVS et actions des AVS, entre les personnes Autistes typiques ou atypiques et les personnes Asperger.

P5: au niveau de la méthodologie, au sein de léchantillon de 83 patients, il faudralt préciser combien de patients sont Autistes, Autistes atypiques et Asperger car cette donnée est reprise dans le tableau 2.

P6: une correction est à faire sur le WISC IV quí est une échelle d'efficience intellectuelle qui va jusqư' 16 ans passé.

P6: une correction de frappe est a faire dans la phrase * les analyses des données consistaient en trois PAS *

P 9: les auteurs montrent une absence de significativité entre les comorbidités psychiatriques et * indices de scolarité $\mathrm{b}$, il faudralt préciser quels sont les indices de scolarité en effet les indices du WISC IV ne sont pas des indices de scolarité mais des indices de niveau cognitif et exécutif. D'autre part, il faudrait dans la méthodologie décrire les différentes comorbidités repérées chez cette population et celles retenues pour l'étude, le terme comorbidité est assez large et peut être que celles des personnes autistes typiques ou atypiques sont différentes de celles des personnes Asperger. 
P10 : les auteurs proposent a des indices plus spécifiques s pour permettre une analyse différente et ouvrir la voie vers dautres recherches, peuvent-ils en donner des exemples et approfondir la réflexion.

L'article ast intéressant et apporte des éléments de compréhension et de réflexion sur la scọlarisation des enfants avec un TSA, néanmoins des précisions doivent être apportées sur certains critères (types de comorbidités, indices de scolarisation) et j'encourage les auteurs à aller plus avant dans leurs réflexions et approfondir la discussion.

Enfin je trouve la présentation des résultats statistiques assez confuse, elle mériterait d'eatre rendue plus lisible.

En espérant vous avoir apporté une aide, je me permets de vous souhaiter mes meilleurs voux pour cette nouvelle année 2014.

Très cordialement 


\title{
L'ENCEPHALE
}
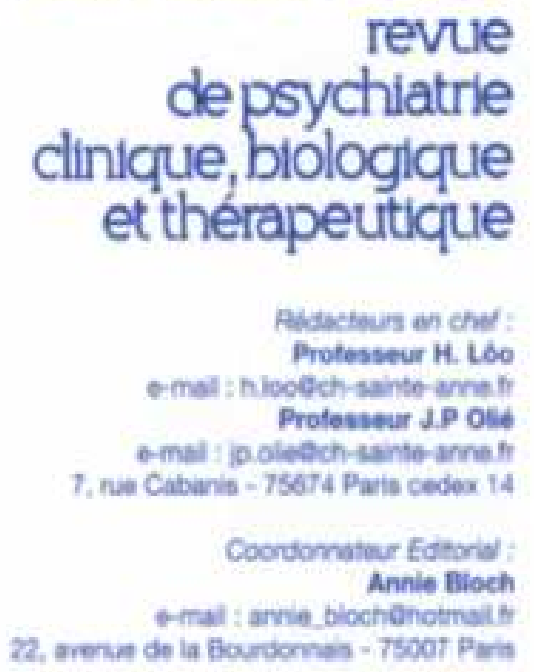

Aldacteurs an cher: Protesseur H. Lo o-mall : Aloogeh-sainte-arne fy Protesseur J.P OSA o-mal 1 jo olecoch-sainte-anne fy 7, nae Cabunis - 75674 Pais cedex 14 Coordomates Edtoris Annie Bioch e-mal s arvie biochenotmal th 2., arenat de la Bourdornas - 7500t Parts

\author{
Mme le Docteur Andrea PIERO \\ Centre Hospitalier Alpes lère \\ Comite de Soutien a 4 Recherche \\ 3, rue de la Gare \\ 38120 SAINT EGREVE
}

$\mathrm{HL} / \mathrm{kg}$

Ref: $2013 / 103$

Paris,

Le 16 Juin 2014

Mudame et chìre Collegue,

J'i le plaisir de vous faire savoir que vore article : La scolarisation des patients atteints par des Troubles du Spectre Autistique sans retard mentalo proposé a l EEncíphule a étée accepté par les réferes pour publication et je vous en feflicite.

Le débi actuel entre la remise i l'éditeur et la pantion d'un article dans rEncíphale est de Pootdre de $9 \mathrm{~d} 12 \mathrm{mos}$.

Dorénavant les articles acceptis dans la revue TEncéphale sont en principe mis en ligne trois semuines après les bons à tirer définitifs et diffués sur ke site de Science Dirnct d'Elevevier Misuon.

Pensez a citer ce texte en biblographie lonque vous terinz sur ce sujet afin de valoriser vore travail et Egalement à adresser un tire a pan aux collegues intéresses par ce sujet.

Je vous prie de croire, Madame et chère Collégue, en lassurance de mes sentiments bien dévouts.

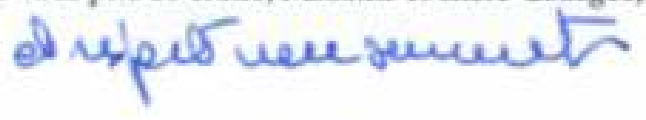

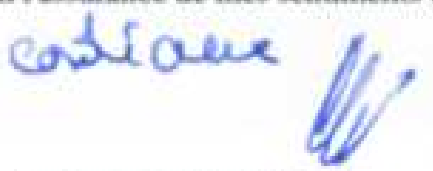

Professeur Henri LOO 


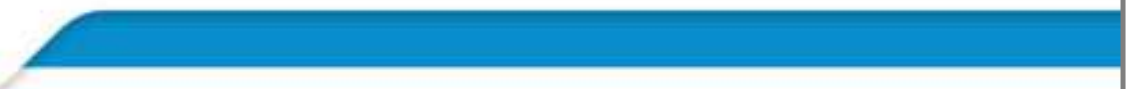

RÉCEPISSÉ

DECLLARATION NORMALE

Numero de declaration

1692118 v 0

du 07-08-2013
MonsieurMARIIOTTI Pgscal

CENTRE HOSPITALIER ALPES ISERE

3 RUE DE LA GARE

BP 100

38120 SAINT EGREVE

\section{[णrgघn}

Nom: CENTREHCSPITAUER ALPES ISERE

Service : COMITEDE SOUTIEN A LA RECHERCHE

Adresse : 3 RUE DE LAGARE BP 100

Code postal : 38120

Ville: SAINT EGREVE
N $=$ SIREN ou SIRET:

26380021100017

Code NAF ou APE :

$8610 Z$

Tél. : 0476564676

Fax. : 0476564525

\section{Traitement déclaré}

Finalité - LOEJECTIF PRINCRAL DE CETTE ETUDE EST D ANALYSER LES LUNS ENTRE LES TROUGLES DU SPECTRE AUTISTIQUE-SANS RETARD MENTAL (TSA-SFM), LES COMORBIDITES PSYCHIATRIQUES ET LA SCOLARTIE LOBJECTIF SECONDARE EST DANALYSER LES FACTEURS COGNTIFS ET CONTEKTUELS LIMITANT LA SCOLAFISATION DE CES ENFANTS ATTEINTS DE TSA-SRM.

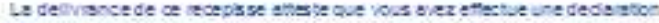
de vole tis: de

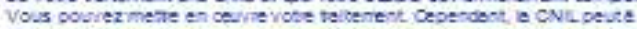

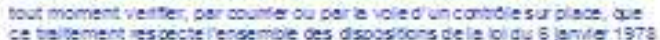

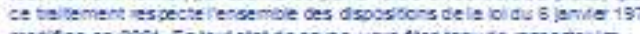

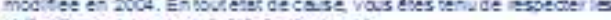

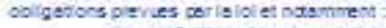

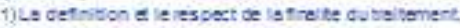

2l la pervence des domets batets

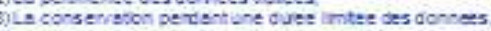

4) La secithe ex a contidenteliedes donnes:

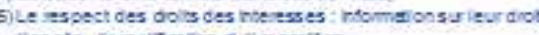

d socts, de iectilicason ed copostion

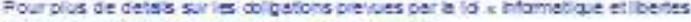

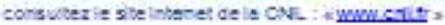

Fait a Paris, le 7 acit 2013

Par delegation de la commis sion

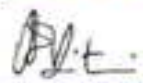

Isabele FALQUE PIERROTN

Presidente 


\section{Bcholalte immédiate.}

Cet item concerne la répéfition immédiale par l'enfant du dernjer éthoncé ou de séries d'énoncés produits par le parent ou l'exnminateur. Lors de la cotation, exclure les répétitions qui sont une amorce de réponse à l'examinateur' on quti sont utillsées comme moyen mnéstque dans des tîches spécifiques

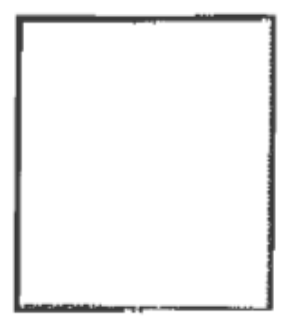

$0=$ Répèle rarement ou jamaís le discours des nulres.

l = Enoncé occassionnellemene écholalique.

$2=$ Répèle des mols et des phrases régultièrement, mais utillise du langage spontané (celui-ci peut t̂tre sléréotypé).

$3=$ Le langage consiste largement en écholalle inmiédiate. 


\section{INTÉRÊTS SENSORIELS INHABITUELS}

CEUX-CI SONT DÉFINIS COMME DES RÉACTIONS PARTICULIËREMENT FORTES OU RÉPÉTÉES OU LA RECHERCHE DE STIMULATIONS RELATIVES AUX 5 SENS DE BASE (LA VUE, LE TOUCHER, L'OUÏE, L'ODORAT OU LE GOÚT), SENSATIONS RECHERCHÉES POUR ELLESMÊMES ET DISSOCIÉES DE LEUR SIGNIFICATION. ON ÉVALUE JUSQU'À QUEL POINT L'INTERÊT ANORMAL PERTURBE OU REMPLACE «L'UTILISATION NORMALE»DE L'OBJET.

Est-ce qu'il/elle semble particulièrement intéressé par la vue, le toucher, le son, le goût ou l'odeur des choses ou des gens? Par exemple, est-ce qu'il/elle a tendance à renifler les jouets, les objets ou les gens de manière inappropriée? Ou est-ce qu'il/elle s'intéresse de façon inhabituelle à la texture des objets ou à leur contact? Ou fixe-t-il/elle des objets ou des lumières pendant de longs moments? Ou porte-til/elle les choses à ses lèvres ou sur sa langue pour tester leur goût ou leur contact? Combien de temps cela a-t-il duré? (NOTER DES EXEMPLES, SPÉCIFIER LE DOMAINE AUDITIF, VISUEL. OLFACTIF, TACTILE.) $\mathrm{Y}$ a-t-il eu dans le passé une époque où il/elle semblait particulièrement intéressé(e) par ces sensations? (NOTER DES EXEMPLES)

INTÉRÊTS SENSORIELS INHABITUELS (DOIVENT AVOIR DURE AU MOINS 3 MOIS)

$0=$ aucun

1 = une ou deux modalités d'intérêt sensoriel inhabituel apparaissant

ACTUELLEMENT

régulièrement

2 = intérêt sensoriel inhabituel occupant beaucoup de temps ou limitant/empêchant ses possibilités d'utiliser le matériel dans

ACTUELLEMENT

sa fonction normale

OU DANS LE PASSÉ

9 = inconnu ou non demandé 

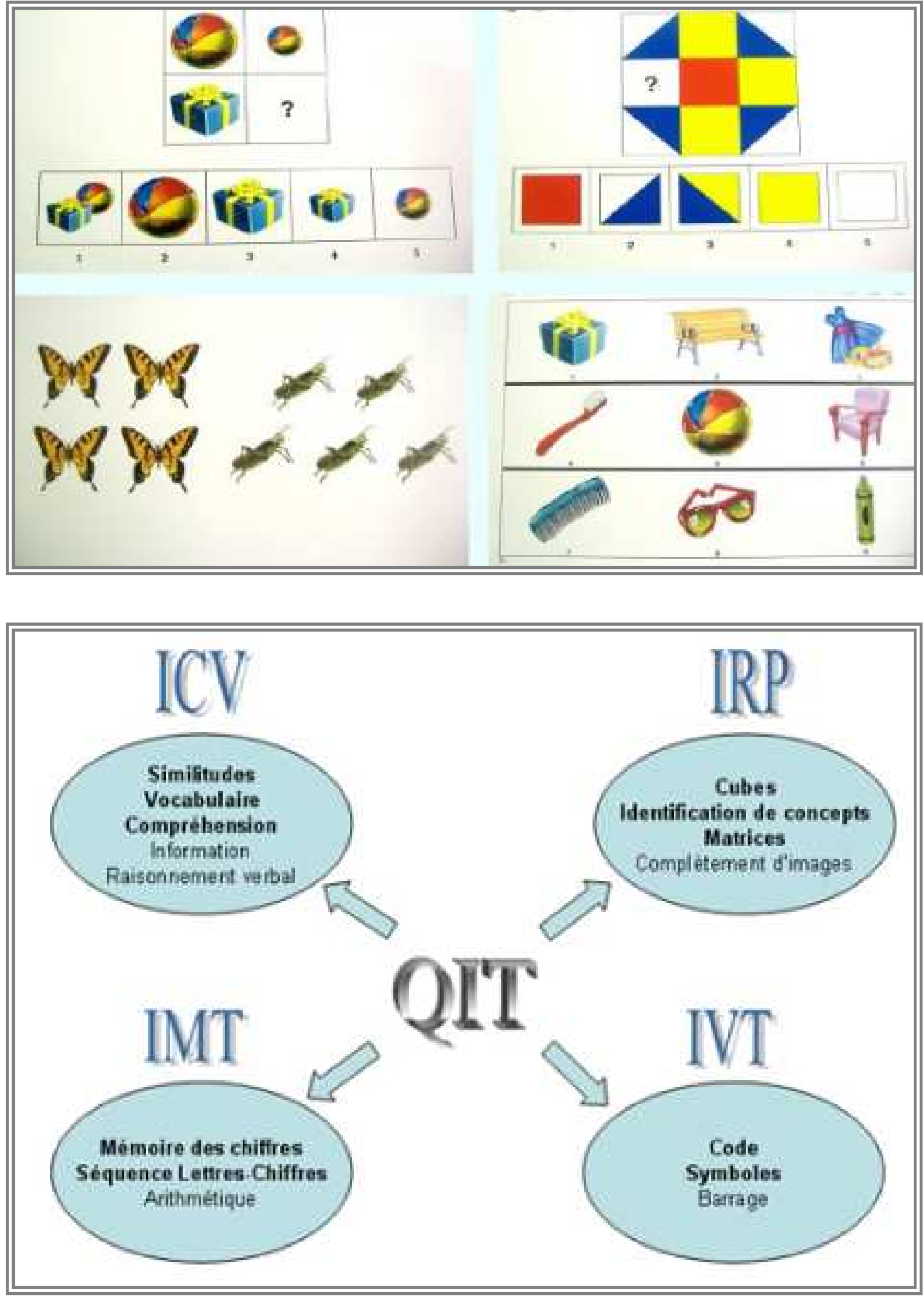


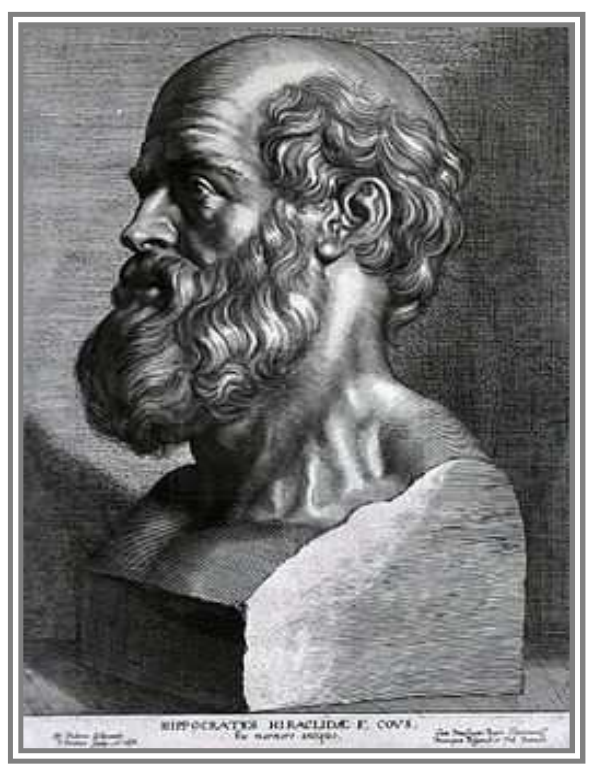

En présence des Maîtres de cette Faculté, de mes chers condisciples et devant l'effigie d'HIPPOCRATE,

Je promets et je jure d'être fidèle aux lois de l'honneur et de la probité dans l'exercice de la Médecine.

Je donnerai mes soins gratuitement à l'indigent et n'exigerai jamais un salaire au dessus de mon travail. Je ne participerai à aucun partage clandestin d'honoraires. Admis dans l'intimité des maisons, mes yeux n'y verront pas ce qui s'y passe; ma langue taira les secrets qui me seront confiés et mon état ne servira pas à corrompre les mœurs, ni à favoriser le crime.

Je ne permettrai pas que des considérations de religion, de nation, de race, de parti ou de classe sociale viennent s'interposer entre mon devoir et mon patient.

Je garderai le respect absolu de la vie humaine.

Même sous la menace, je n'admettrai pas de faire usage de mes connaissances médicales contre les lois de l'humanité.

Respectueux et reconnaissant envers mes Maîtres, je rendrai à leurs enfants I'instruction que j'ai reçue de leurs pères.

Que les hommes m'accordent leur estime si je suis fidèle à mes promesses.

Que je sois couvert d'opprobre et méprisé de mes confrères si j'y manque. 